\title{
Evaluation and comparison of oral function after resection of cancer of the upper gingiva in patients who underwent reconstruction surgery versus those treated with a prosthesis
}

\author{
Yu Ohashi ${ }^{1,3^{*}}$, Kiyoto Shiga ${ }^{2,3}$, Katsunori Katagiri ${ }^{2,3}$, Daisuke Saito ${ }^{2,3}$, Shin-ichi Oikawa ${ }^{2,3}$, Kodai Tsuchida ${ }^{2,3}$, \\ Aya Ikeda ${ }^{2,3}$, Jun Miyaguchi ${ }^{2,3}$, Takahiro Kusaka ${ }^{2,3}$ and Hiroyuki Yamada ${ }^{1,3}$
}

\begin{abstract}
Background: We retrospectively analyzed the articulation, mastication, and swallowing function of patients who underwent reconstruction or used a prosthesis after resection of the upper gingiva.

Methods: This study included patients who underwent resection of cancer of the upper gingiva from January 2014 to December 2018. Articulatory function was evaluated with Hirose's conversational function evaluation criteria. Mastication function was evaluated with the Yamamoto's occlusion table. Swallowing function was assessed with the MTF (Method of intake, Time, Food) score.

Results: The mean articulatory function score was 8 points in the Reconstruction Surgery Group (RSG) and 8.8 points in the Prosthesis Group (PG). The mean mastication function score was 2.8 points in the RSG and 3.3 points in the PG. The mean swallowing function score was M3T4F4 in the RSG and M4T4F4.3 in the PG.
\end{abstract}

Conclusions: The prosthesis depends on the remaining occlusal support area. Our study suggest that prosthesis is better indication when there is more than one occlusal support area.

Keywords: Oral cancer, Neoplasms, Gingival, Maxillofacial surgery, Free flaps, Maxillofacial prosthesis, Palatal obturator

\section{Background}

When radical resection for advanced cancer of the upper gingiva creates an oronasal or oroantral fistula, patients experience dyslalia, dysmasesis, and dysphagia. The quality of life of these patients is impaired because of eating difficulties caused by dysmasesis and dysphagia. Dyslalia results in social difficulty; difficulty with speech and eating can result in avoidance of social situations [1]. To

\footnotetext{
*Correspondence: yohashi@iwate-med.ac.jp

${ }^{1}$ Division of Oral and Maxillofacial Surgery, Department of Oral and Maxillofacial Reconstructive Surgery, Iwate Medical University, Morioka, Japan

Full list of author information is available at the end of the article
}

solve these problems, we generally perform a free flap and/or bone flap or apply a prosthesis after resection of carcinoma of the upper gingiva.

In many cases, a free fibula flap, free scapula flap, or free iliac flap is applied for reconstruction after resection of advanced gingival cancer [2]. Maxillary reconstruction with free flap provides abundant tissue for reconstruction, the freedom to orient, shape, and the ability for reconstruction to be performed as a single stage procedure [3]. Immediate reconstruction surgery has the advantage that the oral-to-maxillary and oralto-nasal-cavity defects are closed in a single operation. Reconstruction with free bone flaps has advantages 
for masticatory function if an implant can be placed. However, disadvantage of flee flaps include longer surgical and recovery times with increased potential for complications compared with prosthetic obturation [4]. And many patients do not desire this treatment because of the increased burden of autologous tissue collection and the necessity of implant placement surgery to improve masticatory function.

Prosthesis has been common approach for treating maxillectomy defects. The advantage of this technique includes a shorter operation time, shorter postoperative hospital stay, and complete visualization of the maxillectomy cavity, which simplifies oncologic surveillance [5]. However, the prosthesis often takes several months to complete its shape and if there is no tooth to support the prosthesis, the prosthesis will not be stable and may not improve chewing function. And larger defects are harder to obturate as the prosthesis may be overly heavy and difficult or impossible to retain, particularly in partially or totally edentulous patients $[6,7]$.

Both methods have drawbacks. However, few reports have compared functional outcomes of free flaps and prostheses, and the choice of reconstruction method remains controversial $[4,8-11]$. Therefore, in this pilot study we evaluated masticatory function, swallowing function, and articulation function after reconstruction performed with a free flap or a prosthesis for advanced maxillary cancer to determine criteria for method selection.

\section{Methods}

Informed consent was obtained from all subjects. This study was approved by the Human Research Ethics Committee at the Iwate Medical University (ethics ID MH2020-210).

Table 1 Hirose's conversational function evaluation criteria

\begin{tabular}{lll}
\hline & (A)family & (B)other people \\
\hline 1. Can understand well & 5 points & 5 points \\
2. Sometimes does not know & 4 points & 4 points \\
3. Can understand if he/she knows a topics & 3 points & 3 points \\
4. Can sometimes understand & 2 points & 2 points \\
5. Can not understand at all & 1 point & 1 point \\
A+B & & \\
Excellent: $10-8$ points can talk everyday, talk on new topics & & \\
MODERATE: $7-5$ points conversation is possible if the topic is limited & & \\
Poor: $\leq 4$ social language life is difficult &
\end{tabular}

\section{Patients}

Twelve consecutive patients who underwent resection of cancer of the upper gingiva followed by reconstruction with a free flap (skin flap and/or bone flap) or with prosthesis application from January 2014 to December 2018 were included in this study. Resection surgery resulted in oronasal or oroantral fistula in all patients. Although reconstructive surgery is the primary method of reconstruction after resection, it was decided to apply a prosthesis to the post-resection reconstruction method with the aim of regressing the operative time in the case of older age or complications of underlying disease.

\section{Methods}

Maxillary defects were classified according to a maxillectomy classification [12] and the occlusion area after resection was classified according to Eichner's Index [13].

Articulatory function was evaluated with Hirose's conversational function evaluation criteria [14], which evaluate the patient's ability to speak with family members and others. The attending physician conducted a questionnaire-guided interview with the patient and evaluated the patient on the basis of the results. This method uses a 5 -point scale. Evaluation points from conversations with family (1-5 points) and from conversations with others who are not family members (1-5 points) are summed. Conversation function is evaluated according to the total score as excellent ( $8-10$ points), moderate ( $5-7$ points),

Mastication function was evaluated with the chewing efficiency judgment table (Yamamoto's occlusion table) [15]. In this evaluation method, patients identify foods they can consume from a list, and the class of the relevant food is scored. Thirty-three types of food, including Japanese foods, are classified into six levels from 1 to 6 , and the class of consumable foods is scored. Soup that can be consumed with an edentulous jaw is 1 point, whereas hard foods have a high score. If a high-scoring food can or poor ( $\leq 4$ points) (Table 1$)$.

Hajime Hirose, Guideline of Head and Neck Cancer. Japan Society for Head and Neck Cancer, 3rd edition, 169, 2018 
be consumed, chewing ability is high. The level at which mastication is possible is evaluated in a stepwise manner according to the chewing efficiency judgment table. The score of the level at which there is a food that can be consumed on the chewing efficiency judgment table is used for evaluation (Table 2).

Swallowing function was assessed with the MTF classification [16]. This evaluation method allows easy evaluation of swallowing function in daily clinical settings. This method evaluates which foods and how much the patient can actually consume. The total score for the three items of Method of intake (M), Time (T), and Food (F) is calculated. Each evaluation item is scored from 1 to 5 points. Ingestible foods are categorized from $\mathrm{A}$ to $\mathrm{E}$, then the number of food categories ingested is summed (Table 3).

Results of each evaluation method were used to compare the reconstruction surgery group (RSG) and the prosthesis group (PG). Statistical analyses were carried out using Mann-Whitney test.

\section{Results}

This study included six men and six women; the mean age of patients was 65.8 years. T classifications included cT2 in three patients (25.0\%), cT3 in one patient (8.3\%), and cT4a in eight patients (66.7\%). The operative procedure for the primary lesion was partial resection in eight patients (66.7\%), subtotal resection in three patients $(25.0 \%)$, and total resection in one patient (8.3\%). The surgical procedure for reconstruction was fibula free flap in two patients (16.7\%) and combined fibula free flap and anterolateral thigh free flap in two patients (16.7\%). A prosthesis was applied in eight patients $(66.7 \%)$.

Defect classification [12] in the RSG was $2 b$ in one patient (8.3\%) and $2 \mathrm{c}$ in three patients (25.0\%). Defect classification in the PG was $2 \mathrm{a}$ in all eight patients (66.7\%).

Eichner's Index [13] scores in the RSG were B2 in one patient (8.3\%), B3 in one patient (8.3\%), C2 in one patient $(8.3 \%)$, and $\mathrm{C} 3$ in one patient $(8.3 \%)$. Eichner's Index scores in the PG were B1 in one patient (8.3\%), B2 in two patients (16.7\%), B3 in two patients (16.7\%), B4 in one patient (8.3\%), and C2 in two patients (16.7\%) (Table 4).

The mean articulatory function score was 8 points (min: 2, max: 10) in the RSG and 8.8 points in the PG; this difference was not significant $(p=0.29)$ (Fig. 1).

The mean mastication function score was 2.8 points (min: 1, max: 6) in the RSG and 3.3 points in the PG. This difference was not significant $(p=0.60)$ (Fig. 2).

The mean swallowing function score was M3T4F4 (min: M1T0F1, max: M5T5F5) in the RSG and M4T4F4.3 in the PG. In the category of food-intake method, patients in the PG ingested in a significantly more normal fashion than those in the RSG $(p=0.04)$. However, there

Table 2 Yamamoto's occlusion table

\begin{tabular}{ll}
\hline Score & Foods \\
\hline 1 & Soup \\
2 & Boiled rice, pudding, tofu \\
3 & Rice, boiled fish, fish mince, tuna sashimi, skewers of eel \\
4 & Steamed rice, roll boiled fish paste, kon-nyaku, sausage, ham, squid sashimi \\
5 & Salami, beef steak, French bread, dry squid, millet brittle, scallop string, \\
& picked scallion, jellyfish vinegar, sea cucumber, vinegar dumpling, \\
6 & shellfish \\
\hline
\end{tabular}

Yamamoto T. The posterior artificial teeth position used for complete dentures cross-bite case. Practice in Prosthodontics 1972;5:395-400, Modification

Table 3 MTF score

\begin{tabular}{|c|c|c|c|c|}
\hline 1. Method of intake & & 2. Time of intake & & 3. Foods \\
\hline Only tube feeding & 1 point & Tube feeding & Nothing & A: water, tea \\
\hline Combination tube feeding & 2 points & MORE than $50 \mathrm{~min}$ & 1 point & B: potage, rich liquid food \\
\hline Invention of meal & 3 points & $40 \mathrm{~min}$ & 2 points & C: jelly, paste food \\
\hline Some restrictions & 4 points & $30 \mathrm{~min}$ & 3 points & D: whole rice bowl, soft food \\
\hline \multirow[t]{2}{*}{ No limit } & 5 points & $20 \mathrm{~min}$ & 4 points & E: regular diet \\
\hline & & $10 \mathrm{~min}$ & 5 points & $\begin{array}{l}\text { *Score the number of food } \\
\text { groups available }\end{array}$ \\
\hline
\end{tabular}

Yasushi Fujiwara, et al. Journal of Otolaryngology of Japan 100: 1401-1407, 1997 
Table 4 Characteristics of the patients

\begin{tabular}{|c|c|c|c|}
\hline Item scales & Total N (\%) & N (\%) of RSG & $N(\%)$ of PG \\
\hline All patients & $12(100)$ & $4(33.3)$ & $8(66.7)$ \\
\hline \multicolumn{4}{|l|}{ Gender } \\
\hline Male & $6(50)$ & $3(25)$ & $3(25)$ \\
\hline Female & $6(50)$ & $1(8.3)$ & $5(60)$ \\
\hline \multicolumn{4}{|l|}{ Age } \\
\hline Mean & 65.8 & 58.5 & 69.5 \\
\hline Range & $41-82$ & $41-71$ & $58-82$ \\
\hline $40-49$ & $1(8.3)$ & $1(8.3)$ & - \\
\hline $50-59$ & $2(16.7)$ & $1(8.3)$ & $1(8.3)$ \\
\hline $60-69$ & $5(60)$ & $1(8.3)$ & $4(33.3)$ \\
\hline $70-79$ & $2(16.7)$ & $1(8.3)$ & $1(8.3)$ \\
\hline $80-89$ & $2(16.7)$ & - & $2(16.7)$ \\
\hline \multicolumn{4}{|l|}{ T classification } \\
\hline $\mathrm{CT2}$ & $3(25)$ & - & $3(25.0)$ \\
\hline сT3 & $1(8.3)$ & - & $1(8.3)$ \\
\hline cT4a & $8(66.7)$ & $4(3.3)$ & $4(3.3)$ \\
\hline \multicolumn{4}{|l|}{ Operation of primary } \\
\hline Limited resection of maxilla & $8(66.7)$ & $2(16.7)$ & $6(50)$ \\
\hline Subtotal resection of maxilla & $3(25.0)$ & $2(16.7)$ & $1(8.3)$ \\
\hline Radical resection of maxilla & $1(8.3)$ & - & $1(8.3)$ \\
\hline \multicolumn{4}{|l|}{ Method of reconstruction } \\
\hline Fibra flap & $2(16.7)$ & $2(16.7)$ & - \\
\hline $\begin{array}{l}\text { Fibra flap }+ \text { Anterolateral } \\
\text { thigh flap }\end{array}$ & $2(16.7)$ & $2(16.7)$ & - \\
\hline Prosthesis & $8(66.7)$ & - & $8(66.7)$ \\
\hline \multicolumn{4}{|l|}{ Defect class } \\
\hline 1 & - & - & - \\
\hline $2 a$ & $8(66.7)$ & - & $8(66.7)$ \\
\hline $2 b$ & $1(8.3)$ & $1(8.3)$ & - \\
\hline $2 c$ & $3(25.0)$ & $3(25.0)$ & - \\
\hline $3 a$ & - & - & - \\
\hline $3 b$ & - & - & - \\
\hline $3 c$ & - & - & - \\
\hline $4 a$ & - & - & - \\
\hline $4 b$ & - & - & - \\
\hline $4 c$ & - & - & - \\
\hline \multicolumn{4}{|l|}{ Eichiner's Index } \\
\hline $\mathrm{B} 1$ & $1(8.3)$ & - & $1(8.3)$ \\
\hline B2 & $3(25)$ & $1(8.3)$ & $2(16.7)$ \\
\hline B3 & $3(25)$ & $1(8.3)$ & $2(16.7)$ \\
\hline B4 & $1(8.3)$ & - & $1(8.3)$ \\
\hline $\mathrm{C} 1$ & - & - & - \\
\hline $\mathrm{C} 2$ & $3(25)$ & $1(8.3)$ & $2(16.7)$ \\
\hline C3 & $1(8.3)$ & $1(8.3)$ & - \\
\hline
\end{tabular}

was no significant difference between groups in intake time $(p=1.00)$ or in food groups $(\mathrm{p}=0.58)$ (Fig. 3$)$.
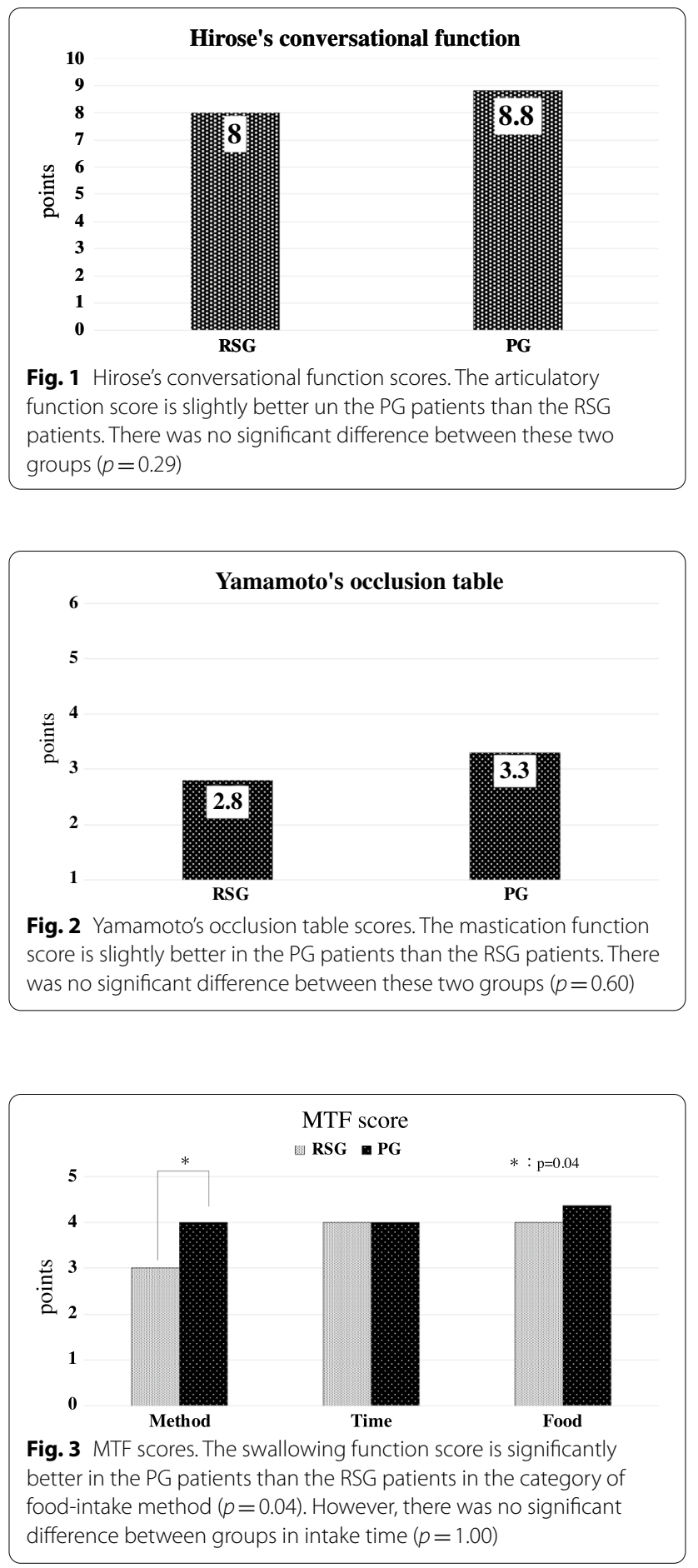

\section{Discussion}

After resection of advanced carcinoma of the upper gingiva, there were two common methods: applying an obturator or performing a reconstructive surgery using a free flap (and/or bone flap). Reconstruction with an obturator has been reported to provide good quality of 
life $[17,18]$. However, obturators can allow food leakage during swallowing [1] and there may be a decrease in feeling of attachment as a result of dry mouth after radiation treatment if the patients underwent postoperate radiotherapy [9]. It is said that prosthesis is not appropriate due to heavy weight $[4,6]$. In contrast, reconstructive surgery using osteocutaneous free flaps (bone flaps) is reported to preserve significant function in the mid-face [19]. This procedure is thought to be highly effective [20], but has the drawback of increasing operation time [4]. In addition, occlusal reconstruction with placement of a dental implant after free bone flap reconstruction lengthens the treatment period, increases the surgical invasiveness, and requires more visits to clinics for treatment. In patients with advanced cancer of the upper gingiva, occlusal reconstruction with a dental implant is ideal after reconstruction of the maxilla with a free bone flap. However, postoperative radiation therapy or radiation chemotherapy is sometimes necessary. In these cases, the patient often does not want the additional visits required after reconstruction surgery. Although each treatment has unique advantages and disadvantages and there have been various reports on each treatment, Maurio et al. [4] reports that extensive maxillectomy defects have better functional outcome with free flap. On the other hand, there are reports that there is no difference in oral function between PG and RSG $[8,10,11]$. Oral functions including mastication, swallowing, and articulation, and maximum restoration of these functions is important. We believe that while aesthetic recovery is important, recovery of oral functions is directly linked to quality of life. Therefore, to clarify whether it is better to apply an obturator or to perform a reconstructive surgery with a free flap, we retrospectively examined the records of patients treated at our facility and evaluated their masticatory function swallowing function, articulatory function and so on. The purpose of this study was to determine treatment selection criteria by evaluating swallowing function and articulation function. Our study indicated that the size of the primary tumor and the occlusal support area influenced the functional evaluation. In this study, the RSG had four cT4a cases, whereas the PG had three cT2 cases, one cT3 case, and four cT4a cases. According to this classification, the PG tended to retain an occlusal support area, which we consider slightly more favorable.

There was little difference between groups in articulatory function, but function tended to be slightly better in the PG than in the RSG. However, some patients with a prosthesis were unable to converse when the prosthesis was removed at night, and a marked decline in function in daily life was a problem. In contrast, patients in the RSG were able to speak at any time, which is an advantage of surgical reconstruction.
Mastication function did not sufficiently recover in either group. It is probable that in the RSG only soft tissue reconstruction was performed in some cases and implant restoration was not performed even in some cases with hard tissue reconstruction. Patients in the PG tended to have good mastication function. If an implant prosthesis can be placed, mastication function is expected to improve. However, this approach increases the number of surgeries and the invasiveness of the procedures. In addition, some patients require prompt surgery to treat the underlying disease and do not have time to carefully consider treatment options. Indications need to be determined based on conditions such as the patient's age, general condition, and the curability of the underlying disease.

With regard to swallowing function, a properly manufactured prosthesis can create a more natural environment in the oral cavity during the oral stage of eating and swallowing. An environment that promotes easy swallowing can be created during the prosthesis preparation process and with fine adjustments after completion. In contrast, with reconstruction surgery, because the formation of the intraoral environment is limited to the intraoperative period, it is difficult to create ideal conditions for restoring swallowing function.

We found no significant difference between the groups in most functional evaluations, although the PG tended to have relatively better functional outcomes. The PG had significantly better results in the method of intake; we think this difference is related to better swallowing function resulting from better marginal fitting of the prosthesis. The RSG did not have ideal occlusal recovery. These results indicate that occlusal reconstruction with a dental implant is necessary for better outcomes after reconstruction surgery. In both groups, masticatory function was markedly reduced. Because use of a prosthesis depends on the remaining occlusal support area, if this area is small, masticatory function may not recover. If a dental implant can be placed, masticatory function is expected to recover. When deciding whether to perform reconstruction surgery or to apply a prosthesis, it is important to focus on the occlusal support area after resection as well as the maxillary defect area, to consider the advantages and disadvantages of each method, and to fully consult with the patient and related medical staff. In patients with advanced maxillary cancer who do not have much time to prepare for surgery and in environments where reconstructive surgery is not possible, a prosthesis may be considered as a first choice. Our study suggest that prosthesis tend to have better oral function for Eichner B3. In addition, if radical resection is possible, patients understand the procedure, and there is no problem 
with patient age, underlying disease, or waiting time for surgery, reconstructive surgery and functional restoration with implant prosthesis is preferable.

A limitation of this study was due to the study design. This pilot study was a retrospective review of small number of the patients who underwent maxillectomy in our institutions. Therefore, an organized prospective study is needed to evaluate the treatment results of the patients who undergo maxillectomy. If possible, multi-institutional prospective study is necessary to clarify the criteria of the selection of functional treatment for the patients who undergo maxillectomy.

\section{Conclusions}

There was no significant difference between the two treatment groups in most functional evaluations, although the PG tended to have relatively better outcomes than the RSG. The PG had significantly better results in the method of food intake. This finding may be related to better swallowing function resulting from better marginal fitting of the prosthesis. Occlusal reconstruction with a dental implant is necessary to improve outcomes after reconstruction surgery. Our study suggest that prosthesis is indicated for Eichner B3. If radical resection is possible, the patient understands the procedure, and there are no problems with patient age, underlying disease, or waiting time for surgery, reconstructive surgery and functional restoration with implant prosthesis is preferable.

\section{Abbreviations}

RSG: Reconstruction Surgery Group; PG: Prosthesis Group.

\section{Acknowledgements}

This work was supported by JSPS KAKENHI Grant Number 20K10251. We thank Rebecca Tollefson, DVM, from Edanz Group (https://en-author-services.edanz group.com/) for editing a draft of this manuscript.

\section{Authors' contributions}

$\mathrm{YO}$ and $\mathrm{KS}$ wrote the main manuscript text. KK, DS, SO, KT and Al prepared Figs. 1,2and3. HY, JM and TK prepared Tables 1,2,3and4. All authors read and approved the final manuscript.

\section{Funding}

This research did not receive any specific grant from funding agencies in the public, commercial, or not-for-profit sectors.

\section{Availability of data and materials}

All data used and/or analyzed during this research are available from the corresponding author on reasonable request.

\section{Declarations}

\section{Ethics approval and consent to participate}

Informed consent was obtained from all subjects. All participants received information about the study and agreed to participate by submitting a questionnaire. This study was approved by the Human Research Ethics Committee at the Iwate Medical University (ethics ID MH2020-210). This study was conducted according to the principles of the Declaration of Helsinki.

\section{Consent for publication}

Not applicable.

\section{Competing interests}

The authors declare that they have no competing interests.

\section{Author details}

${ }^{1}$ Division of Oral and Maxillofacial Surgery, Department of Oral and Maxillofacial Reconstructive Surgery, Iwate Medical University, Morioka, Japan. ${ }^{2}$ Department of Head and Neck Surgery, I wate Medical University, Yahaba, Japan. ${ }^{3}$ Head and Neck Cancer Center, Iwate Medical University Hospital, Yahaba, Japan.

Received: 28 February 2021 Accepted: 7 July 2021

Published online: 15 July 2021

\section{References}

1. Irish J, Sandhu N, Simpson C, Wood R, Gilbert R, Gullane P, et al. Quality of life in patients with maxillectomy prostheses. Head Neck. 2009;31:813-21.

2. Cordeiro PG, Bacilious N, Schantz S, Spiro R. The radial forearm osteocutaneous "sandwich" free flap for reconstruction of the bilateral subtotal maxillectomy defect. Ann Plast Surg. 1998;40:397-402.

3. Shestak KC, Schusterman MA, Jones NF, Johnson JT. Immediate microvascular reconstruction of combined palatal and midfacial defects using soft tissue only. Microsurgery. 1988;9:128-31.

4. Moreno MA, Skoracki RJ, Hanna EY, Hanasono MM. Microvascular free flap reconstruction versus palatal obturation for maxillectomy defects. Head Neck. 2010;32:860-8.

5. Davison SP, Sherris DA, Meland NB. An algorithm for maxillectomy defect reconstruction. Laryngoscope. 1998;108:215-9.

6. Okay DJ, Genden E, Buchbinder D, Urken M. Prosthodontic guidelines for surgical reconstruction of the maxilla: a classification system of defects. J Prosthet Dent. 2001;86:352-63.

7. Futran ND, Wadsworth JT, Villaret D, Farwell G. Midface reconstruction with the fibula free flap. Arch Otolaryngol Head Neck Surg. 2002;128:161-6.

8. Rogers SN, Lowe D, McNally D, Brown JS, Vaughan ED. Health-related quality of life after maxillectomy: a comparison between prosthetic obturation and free flap. J Oral Maxillofac Surg. 2003;61:174-81.

9. Kreeft AM, Krap M, Wismeijer D, Speksnijder CM, Smeele LE, Bosch $S D$, et al. Oral function after maxillectomy and reconstruction with an obturator. Int J Oral Maxillofac Surg. 2012;41:1387-92.

10. Eckardt A, Teltzrow T, Schulze A, Hoppe M, Kuettner C. Nasalance in patients with maxillectomy defects: reconstruction versus obturation. J Craniomaxillofac Surg. 2007;35:241-5.

11. Rieger JM, Tang JA, Wolfaardt J, Harris J, Seikaly H. Comparison of speech and aesthetic outcomes in patients with maxillary reconstruction versus maxillary obturators after maxillectomy. J Otolaryngol Head Neck Surg. 2011:40:40-7.

12. Brown JS, Rogers SN, McNally DN, Boyle M. A modified classification for the maxillectomy defect. Head Neck. 2000;22:17-26.

13. Eichner K. Uber eine Gruppeneinteilung der Luckengebisse fur die Prothetik. Dtsch Zahnarztl Z. 1955;10:1831-4.

14. Takakuni K, et al. General rules for clinical studies on head and neck cancer. 5th ed. Tokyo: Japan Society for Head and Neck Cancer; 2012. p. 76.

15. Toshiyuki I, et al. General rules for clinical and pathological studies on oral cancer: a Synopsis. Jpn J Clin Oncol. 2012; 42:1099-1109

16. Fujimoto Y, et al. Swallowing ability scale for oral and oropharyngeal cancer patients. J Otolaryngol Jpn. 1997;100:1401-7.

17. Chigurupati R, Aloor N, Salas R, Schmidt BL. Quality of life after maxillectomy and prosthetic obturator rehabilitation. J Oral Maxillofac Surg. 2013;71:1471-8. 
18. Kornblith AB, Zlotlow IM, Gooen J, Huryn JM, Lerner T, Strong EW, et al. Quality of life of maxillectomy patients using an obturator prosthesis. Head Neck. 1996;18:323-34.

19. Joseph ST, Thankappan K, Buggaveeti R, Sharma M, Mathew J, Iyer S. Challenges in the reconstruction of bilateral maxillectomy defects. J Oral Maxillofac Surg. 2015;73:349-56.

20. Cao Y, Yu C, Liu W, Miao C, Han B, Yang J, et al. Obturators versus flaps after maxillary oncological ablation: a systematic review and best evidence synthesis. Oral Oncol. 2018;82:152-61.

\section{Publisher's Note}

Springer Nature remains neutral with regard to jurisdictional claims in published maps and institutional affiliations.
Ready to submit your research? Choose BMC and benefit from:

- fast, convenient online submission

- thorough peer review by experienced researchers in your field

- rapid publication on acceptance

- support for research data, including large and complex data types

- gold Open Access which fosters wider collaboration and increased citations

- maximum visibility for your research: over $100 \mathrm{M}$ website views per year

At BMC, research is always in progress.

Learn more biomedcentral.com/submissions 